\title{
Tennis balls judgment model based on numerical simulation
}

\author{
Feng Li ${ }^{1}$, a Lu Liu ${ }^{1}$, Qiaohui Wang ${ }^{2}$, Kunling Qin ${ }^{3}$, Qiongqiong Hü ${ }^{4}$, Qian Yang ${ }^{1}$, Yanni Liu ${ }^{1}$ and Bing Zhang ${ }^{1, b}$ \\ ${ }^{1}$ Institute of Physical Education, Huanggang Normal University, Huangzhou 438000, China \\ ${ }^{2}$ Macaomiao Primary School, Tuanfeng 438000, China \\ ${ }^{3}$ Yidu Gaobazhou Middle School, Yidu 443300, China \\ ${ }^{4}$ Fangxian Mengu Middle School, Shiyan 442108, China
}

\begin{abstract}
According to the principle of "eagle eye", assuming that in the absence of the wind and air density uniform, tennis because of deformation produced by hitting, accurate to tennis in flight by gravity, air resistance, the tennis movement into the vertical direction and horizontal direction. Solution can be obtained using the ideas of calculus, thus draws the tennis range, fall to the ground, placement time and location. Increase the rotation of the tennis, according to the formula of sports biomechanics, the pressure difference to produce in vertical direction of the velocity and angular velocity magnus force, solving the stead yunus force coefficient, through the best tennis in the vertical direction of the time needed for higher level computation, model are described in detail in tennis trajectories of the rotating cases and placement, and intuitive simulation diagram of the flying track.
\end{abstract}

\section{Introduction}

Tennis event has swept the world, is a kind of traditional elegant event that integrates competitiveness and appreciation. In competitive process, except for referees, it needs multiple linesmen to judge drop point through getting close to line-out. Even so, due to tennis running speed in the air is so fast, after landing, it often has disputes on players' drop point is in line or out of line.

"Hawk-eye" is called instant review system, it utilizes high speed camera to simultaneous capture tennis flight trajectory basic data from different perspectives; then generates the data into three-dimensional images ; finally it utilizes instant imaging technique to clearly presents tennis movement route and drop point. "Hawk-eye" indicated drop point is a shadow, is not achieved by shooting but by precise calculating flight data. Zhao Yang analyzed tennis players drop point anticipation accuracy influence factors, explored high level athletes and low level athletes' tennis drop points' prediction and judgment differences generation factors [1-3]. All above literatures are analyzing from drop point importance, and little literatures are starting from tennis drop point prediction and judgment. According to "Hawk-eye" principle, the paper establishes tennis flight route models in case with rotation, non-rotation, and considering air resistance, judges tennis drop point, utilizes Matlab software to draw intuitional flight trajectory graph [4-6].

\section{Tennis force analysis}

Tennis belongs to one kind of balls events; it has common features of ball kind event. If it ignores air resistance, then in case there is no rotation, only suffers gravity effects, its trajectory is standard parabola. But tennis soaks in the air, air has viscosity, so it has resistance on moving objects, in ball kind event, air resistance has great impacts on ball horizontal displacement, calculate tennis shooting range should consider air resistance effects.

According to fluid mechanics principle [7], air resistance can divide into friction resistance and pressure resistance. For tennis, its surface is not smooth, so it has friction resistance. And due to its movement speed is fast, Reynolds number is larger, then it surely has pressure resistance [4], the two items resistance compounds into air resistance that is always in the opposite direction of tennis movement speed.

Due to coefficient $\omega$ is unknown, it should calculate its size by inputting trajectory passed coordinate point $(0.2215$, $1.5517,1.0485)$, then can define trajectory equation; its trajectory equation is equal to a section of vertical direction's parabola that opens with certain arc. By establishing calculus solved mathematical model, and utilize Matlab software, respectively draw its vertical direction parabola trajectory and horizontal direction deflection trajectory, then it can work out tennis drop point in case it has rotation.

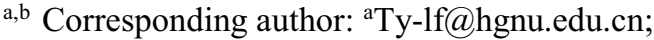
btiyuxi@qq.com
} 


\section{Tennis force flight model establishment}

At first, set one point three-dimensional coordinate as $\left(x_{0}, y_{0}, z_{0}\right)$, speed size as $v_{0}$, direction is $(p, q, 1)$, for the purpose of computation convenience, it can firstly regulate the point as new established coordinate system origin, take speed direction horizontal projection as $\mathrm{x}$ axis direction, $\mathrm{z}$ axis changes into $\mathrm{y}$ axis direction, then tennis speed direction is $\left(\sqrt{p^{2}+q^{2}}, 0,1\right)$. Take new coordinate system as calculation criterion, then tennis movement trajectory changes into two-dimensional figure that is figure in 0-xz plane, decompose speed, from which $\mathrm{f}$ size is in direct proportion to speed $\mathrm{V}$ quadratic, it can be simplified into $f=k v^{2}$.

\section{Matlab numerical simulations}

Make specific quantization on initial values data, it also can make specific quantization on drop point coordinate, similarly, utilize Matlab software, it can accurate describe tennis flight trajectory in the air. In the following, list known quantities one by one:

$$
\begin{aligned}
& h=1 m, m=200 g \\
& k=1 / 2 c \rho A=3.982 \times 10^{-3}, \\
& g=9.8 m / s^{2} \\
& v_{0 x}=0.9749 m / s \\
& v_{0 y}=15.7198 m / s
\end{aligned}
$$

Combine with two intervals, then as following Figure 1 shows:

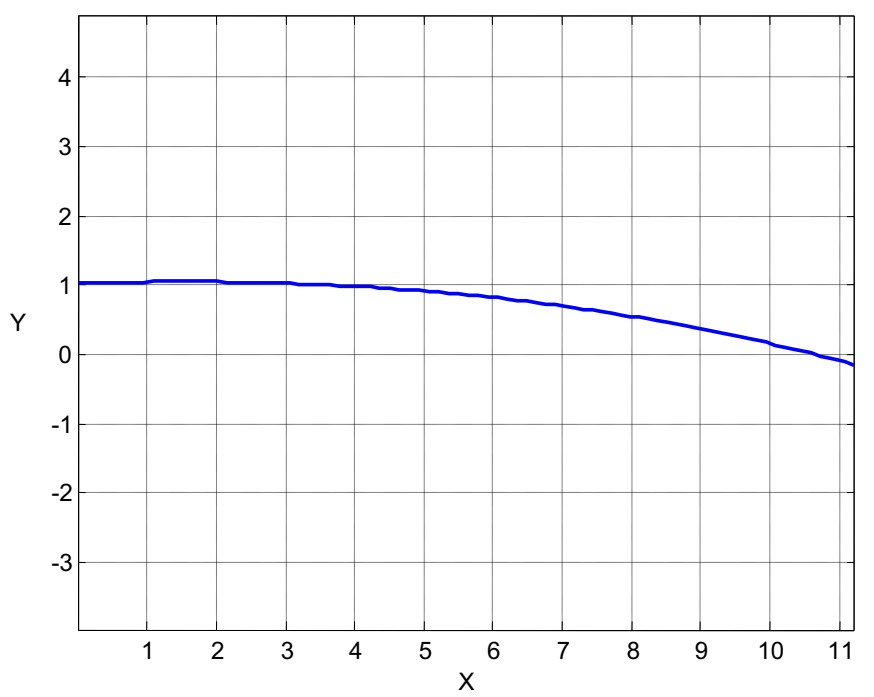

Figure 1. Tennis flight two-dimensional trajectory figure.

Now it needs to transform coordinates back to original coordinates:

$$
x_{t}=x_{0}+x \cdot \frac{p_{0}}{\sqrt{p_{0}^{2}+q_{0}^{2}}}
$$

$$
\begin{gathered}
y_{t}=y_{0}+x \cdot \frac{q_{0}}{\sqrt{p_{0}^{2}+q_{0}^{2}}}, \\
z_{t}=y
\end{gathered}
$$

Then make $\left(x_{t}, y_{t}, z_{t}\right)$ three-dimensional figure as following Figure 2:

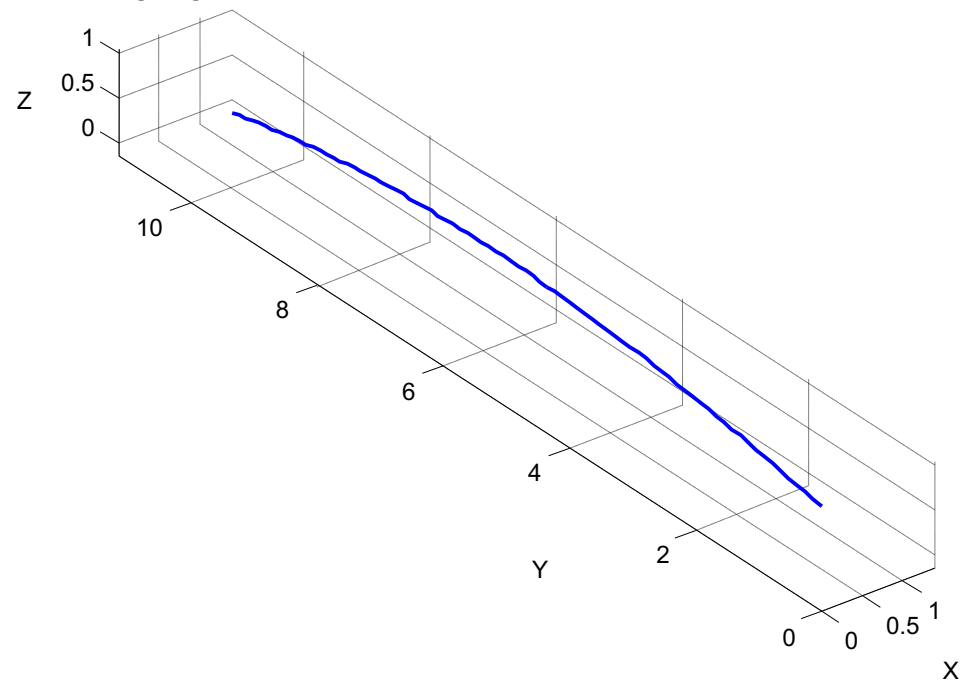

Figure 2. Tennis flight three-dimensional trajectory figure.

Finally, tennis drop point is $(1.5249,12.1991$, and 0$)$.

\section{Model expansions}

In tennis playing process, due to it should generate horizontal deflection, it often exerts rotation so that ball will increase Magnus Force that is vertical to speed direction and angular speed direction in movement, force changes, and trajectory naturally will change, and its flight route is not just in one plane but is a space curve. Magnus force size is in direct proportional to speed size, for convenience, it can be written into $F_{M}=G v, \mathrm{G}$ is related to air density, tennis radius, rotational speed, its size is unknown, so it needs to solve by another coordinate point that trajectory goes through.

For the purpose of calculation convenience, it still needs to transform coordinate system, take speed horizontal projection as $\mathrm{x}$ axis direction, offset direction as $\mathrm{y}$ axis direction, vertical direction $\mathrm{z}$ axis remains unchanged, then after axis transformation, it has following relationship:

$$
\begin{gathered}
x^{\prime}=x \cos \alpha+y \sin \alpha, \\
y^{\prime}=x \sin \alpha-y \cos \alpha, \\
\cos \alpha=\frac{2}{\sqrt{260}}, \\
\sin \alpha=\frac{16}{\sqrt{2^{2}+16^{2}}}=\frac{16}{\sqrt{260}}
\end{gathered}
$$

After coordinates succeed in transformation, then it can list differential equation and then describe tennis.

Due to tennis almost has no displacement in vertical direction, air resistance components in vertical direction 
can be ignored, so, it only suffers gravity impacts in vertical direction, decompose initial time speed, and can get: $v_{z}=0.975 \mathrm{~m} / \mathrm{s}$, in vertical direction, rising $1.0485-1=0.0485 \mathrm{~m}$, it needs time $t^{\prime}=0.0990 \mathrm{~s}$, by calculating, two time phases relative error is only $0.6 \%$, it can be ignored, so assume that tennis rotational axis being parallel to $\mathrm{z}$ axis is at work. After that, transform formula of coordinates: $x^{\prime}=x \cos \alpha+y \sin \alpha, y^{\prime}=x \sin \alpha-y \cos \alpha$

Then solve trajectory equation under original coordinate:

$$
\begin{gathered}
x=5.5896(8-8 \cos 0.3495 t+\sin 0.3495 t) \\
y=5.5896(8 \sin 0.3495 t-1+\cos 0.3495) \\
z=-4.9 t^{2}+0.975 t+1
\end{gathered}
$$

In the following, calculate tennis dropping time $\mathrm{T}$ : $v_{o z} T-\frac{1}{2} g T^{2}=-1$

It can get: $T=0.5621 \mathrm{~s}$. Utilize Matlab [8] to draw flight trajectory in the interval $t \in[0,0.5621]$ as following Figure 3 shows:

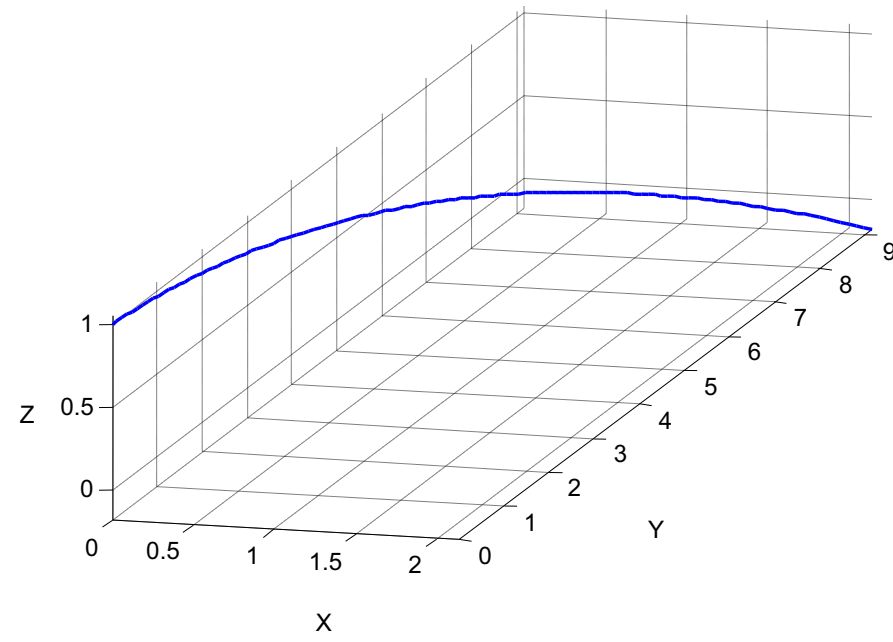

Figure 3. Tennis flight trajectory simulation graph.

\section{.}

Finally, work out tennis drop point as $(1.9474,8.6042$,

\section{Conclusion}

The paper applies integral principle to correctly describe tennis flight speed changes and trajectory curve; it avoids errors that are caused by unchanged speed and other assumptions. In addition, when establish model, we apply coordinate rotation transformation method, it greatly reduces calculation difficulty, and by coordinate transformation, transform three-dimensional figure into two-dimensional figure, it strengthens tennis flight route intuition to a certain angle, but increases steps of transforming coordinates into original coordinates, it needs more rigorous logic. The paper result is more specific and accurate comparing to other literatures.

\section{References}

1. N. Li, J.D. Zhou, Journal of Jilin Institute of Physical Education. 27, 45-47 (2011).

2. X. Wang, Journal of Nanjing Institute of Physical Education. 16, 96-97 (2002).

3. J.L. Yang et al., Journal of Shandong Physical Education Institute. 18, 51-53 (2002).

4. S. Thrun, D. Fox, W. Burgard, F. Dellaert, Artificial Intelligence Journal. 99-41 (2001).

5. L.P. Kan, Bulletin of Sport Science \& Technology. 19, 19-20 (2011).

6. J.X. Zhang, Journal of Hubei Sports Science. 21, 74-75, 79 (2002).

7. Y. Ye, C. Chen, Journal of Coastal Research. 73 577-583 (2015).

8. V. Hariharan, P.S. Srinivasan, Journal of Mechanical Engineering Research and Developments. 37, 42-52 (2014). 